\title{
An ECG Web Services Portal for Standard and Serial ECG Analysis with Enhanced 3D Graphical Capabilities
}

\author{
Jocelyne Fayn ${ }^{1}$, Paul Rubel ${ }^{1}$ \\ ${ }^{1}$ INSERM US7: SFR Santé Lyon-Est, eTechSanté, Université de Lyon, Lyon, France
}

\begin{abstract}
The possibility to remotely use powerful computerized ECG analysis methods at any time is of utmost importance for the early detection of cardiovascular diseases. We present an ECG web services portal that allows to use on request our serial and unary ECG processing methods, CAVIAR and/or the Lyon program.

After authentication, the user can upload digital ECG signal data encoded according to SCP-ECG or in a specific ASCII format. The Lyon program (re-)analyses the ECGs and provides SCP-ECG files containing a typical beat, wave onsets and offsets, and a downloadable table of standard ECG measurements. The CAVIAR web service provides a downloadable table of serial measurements and interactive $3 D$ graphical representations of spatiotemporal QRS and T changes between the typical/median beats of the uploaded ECG records or between the uploaded beats, after their optimized superimposition. As a result, precise measures of ECG changes in amplitude, orientation and duration (QRS, QT ...) are obtained, after correction for wave delineation errors by means of spatiotemporal resynchronization of the compared beats. User-defined or automated comparisons with a reference ECG and/or with the earliest or the previous one can be performed. 3D graphic plots of the superimposed QRS and $T$ pairs are displayed in 1, 2, 4, 6 or 9 panels/page. The user can then rotate each plot, or all plots simultaneously, in any $3 D$ direction, to highlight relevant spatiotemporal changes.

We present the various functionalities of our FR-ECG web portal http://FR-ECG.univ-lyon1.fr and some results.
\end{abstract}

\section{Introduction}

Electrocardiograms are still the main, easy to record everywhere, timely source of information for the diagnosis and the assessment of cardiovascular diseases. Computerized ECG analysis is needed to get reliable ECG measurements for a prompt and efficient physician interpretation. In addition, serial ECG analysis is highly recommended for improving ECG interpretation accuracy
[1]. Thanks to the SCP-ECG standard communications protocol, digital ECGs can be easily stored in any device and quickly transmitted via any type of network (Internet or mobile phone based), in a rich format allowing their reanalysis when needed $[2,3]$. The concomitant extensions of SCP-ECG and of the concept of web services architecture, which provides interoperability facilities and means of transparent access to business applications as well as flexible design tools, gives opportunities to set-up an improved ECG processing web portal.

The aim of this paper is to present our FR-ECG web portal http://FR-ECG.univ-lyon1.fr which allows to remotely access to three ECG processing services based on our well tested unary and serial analysis business applications, the Lyon program [4] and CAVIAR [5-7], each being used either independently or in combination.

The Lyon program provides standard 12-lead ECG measurements and encoded SCP-ECG files embedding the Lyon analysis results with a delineated typical beat and its fiducial points. CAVIAR is an original method of serial ECG analysis aimed at precisely assessing 3D spatiotemporal ECG changes. After the proof of concepts demonstrated and reported by different authors on several databases, the challenge is now to widen the dissemination of the tools and to scale-up their use. ICT technologies and Internet nowadays provide the means required for securely and transparently sharing services and experiences as well as powerful graphical tools which we have implemented for uncovering new findings about spatiotemporal QRS and $\mathrm{T}$ wave changes.

The next section describes the FR-ECG web services architecture and its functional principles. Section 3 will present some reference data on CAVIAR analysis results.

\section{Web services architecture}

FR-ECG allows authenticated access to three types of ECG business processes services: 1) the LYON program for standard ECG analysis, 2) CAVIAR serial ECG analysis for spatiotemporal changes assessment, and 3) CAVIAR analysis combined with the Lyon program for ECG pre-processing. Figure 1 describes the whole architecture and its composition workflow. 

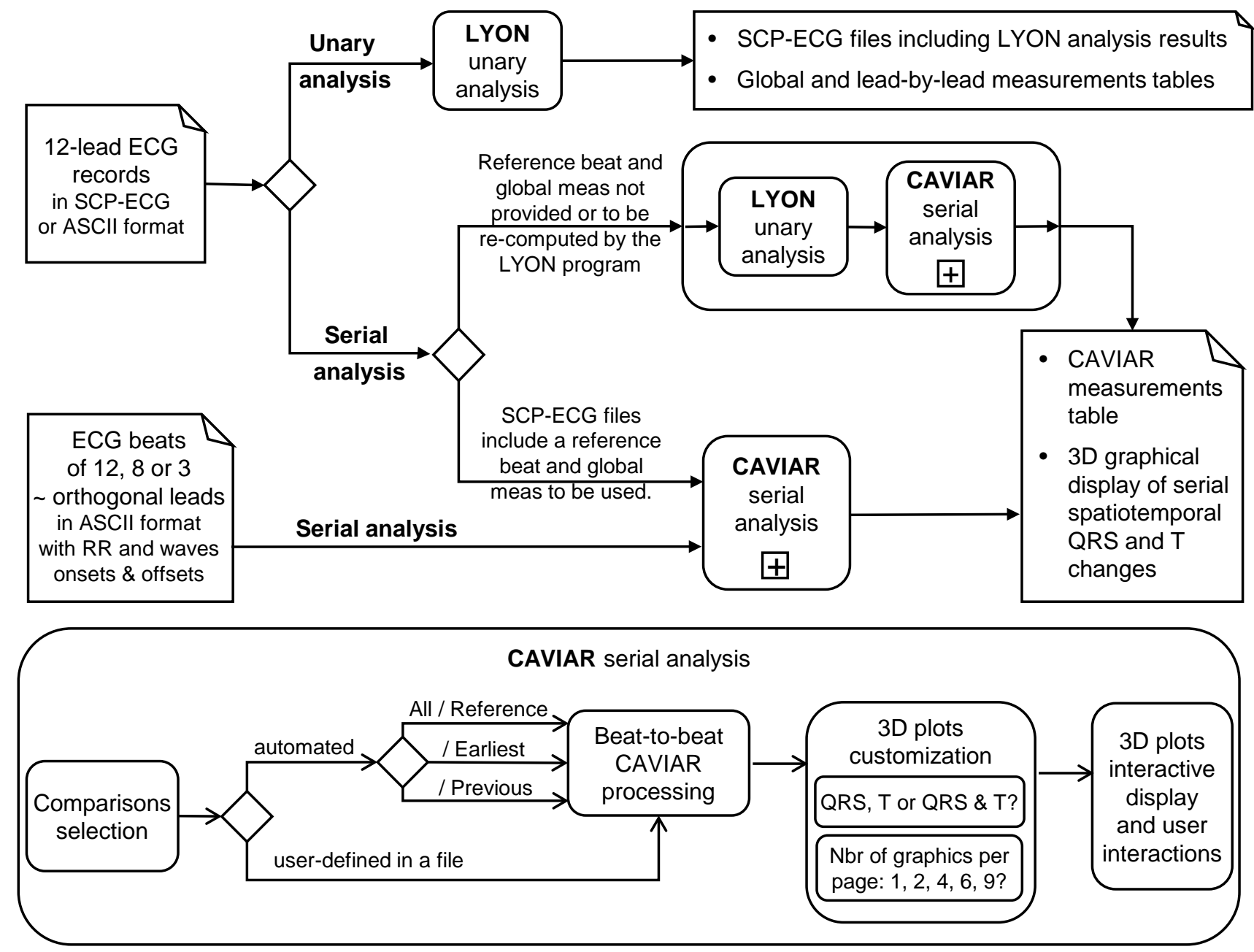

Figure 1. Business processes of the FR-ECG web services architecture with the expanded CAVIAR sub-process.

\subsection{The LYON ECG processing service}

This service has been designed to analyse around 10 second of a standard 12-lead SCP-ECG record, by using the LYON program [4]. The latter typifies the beats of the ECG record and selects a reference beat which is the closest to the dominant class median beat. It computes $\mathrm{P}$, QRS and $T$ onsets and offsets, lead-by-lead and global measurements: PP, RR, PQ, QT, P, QRS and T durations; $\mathrm{P}, \mathrm{QRS}$ and T axes; QTc corrected by the Bazett and by the extensively assessed on different study populations CAVIAR formula (QTc_cav $=$ QT $-(8.7 * \sqrt{R R})+275.1)$. The lead-by-lead measurements include the 22 main amplitude and duration measurements defined in SCP-ECG section 10 "Lead measurement block", plus the QRS peak-to-peak amplitude and integral, and the ST-amplitude at 2/8 and $3 / 8$ of the ST-T (J-point to T-end) wave component.

Input data are a zip file including a set of ECG records, each being encoded either according to the SCP-ECG standard or in a required ASCII format. The LYON ECG service, when used alone, provides at the end of the process a downloadable ecgs_lyon.zip file that includes the original ECG records updated with the LYON processing results encoded as SCP-ECG files and 13 additional files in tabular format respectively containing, for all uploaded ECGs, the global measurements and the measurements for each of the 12 leads, in tables that can be opened with any spreadsheet.

\subsection{The CAVIAR serial analysis service}

The CAVIAR method is a unique method for assessing beat-to-beat spatiotemporal ECG changes [5-7]. It first consists in representing two QRS or T waves from standard 12-lead or pseudo-orthogonal ECGs in their own 3D reference system which is linked to the anatomic axes of the heart and not to the thorax ones as the recorded ECG leads are. This reference system, called (G, UVW) preferential space, is based on the inertia of the electrical forces, $\mathrm{G}$ being their centre of gravity. This representation mode allows to clear beat-to-beat analysis from heart and electrodes position changes. Then, the CAVIAR algorithm 


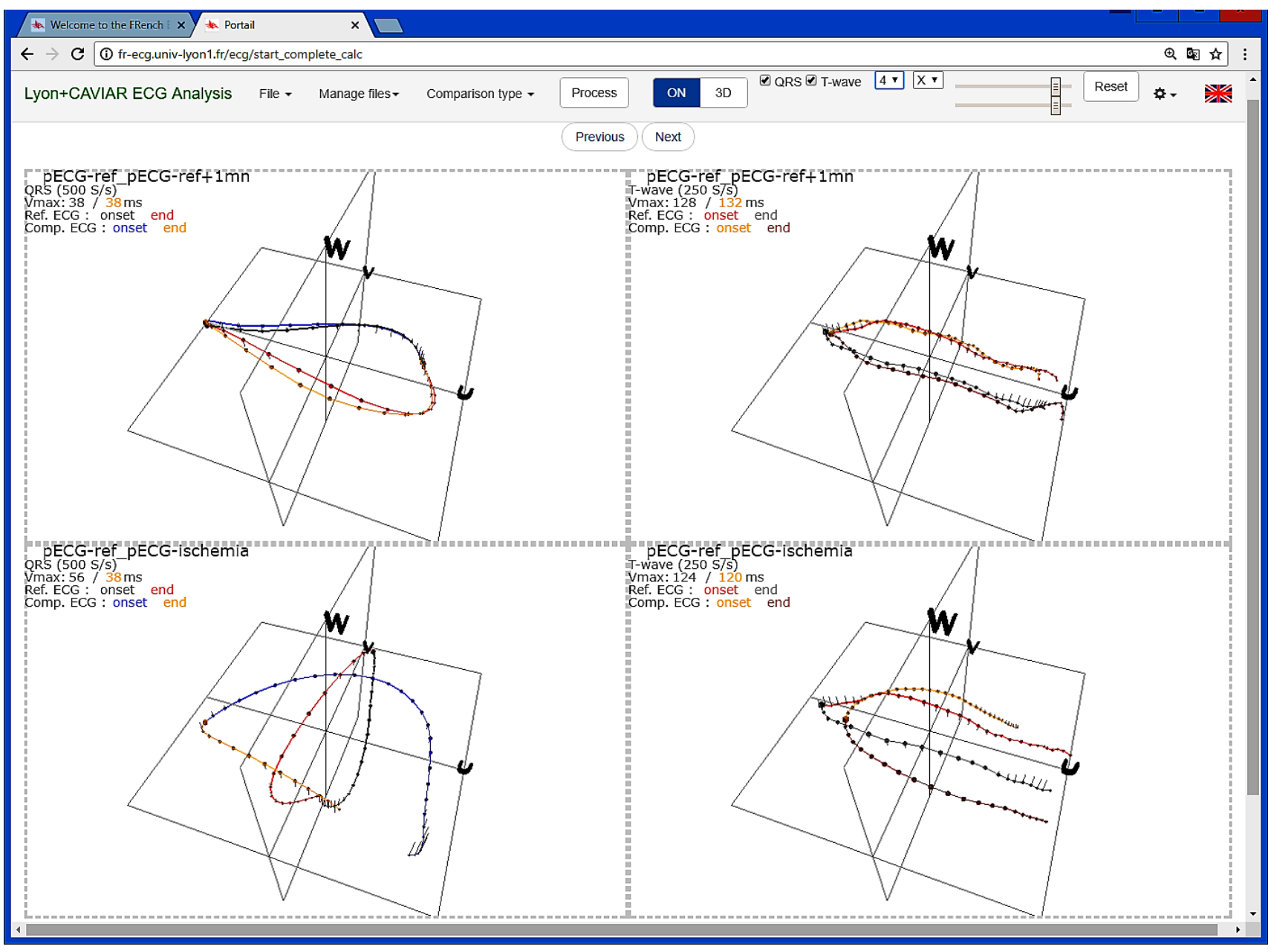

Figure 2. Example of 3D graphical display from the CAVIAR web service, showing serial QRS and T spatiotemporal changes corresponding to ischemic changes.

best superimposes the compared spatiotemporal wave patterns for minimizing their differences by rotation, dilatation and resynchronization, transformations that are invariant to the intrinsic electrical activity of the heart. Thus, temporal and structural ECG changes in amplitude, orientation and duration of cardiac origin are highlighted.

Input data for the CAVIAR analysis service are a zip file including a set of at least 2 ECG records or beats to be compared, each of which being encoded in a file (Fig. 1). Two workflow processes are available: 1) CAVIAR analysis of the uploaded ECGs, based on the provided preprocessing results (reference beat to be used with delineated waves and RR), or 2) CAVIAR analysis of the ECGs after pre-processing by the LYON Program by services composition invocation (explained in the next section). In the first case, the ECG records data must be provided with their own pre-processing results either in the SCP-ECG format or as a set of 12,8 or 3 pseudoorthogonal lead ECG beats in the ASCII format with RR and waves onsets and offsets.

Serial comparisons to be performed can be user-defined in an uploadable Excel file or automated by systematically comparing all the ECGs of each patient with his/her reference, earliest or previous ECG. At the end of the process, CAVIAR provides a set of 28 measurements in a downloadable txt file that is compliant with any spreadsheet. It includes four types of serial measurements: 1) Spatiotemporal changes of QRS (QMQD), of its $~ 30$ first (IMQD) and last (TMQD) ms, and of the Repolarization wave (RMQD); 2) Changes, after optimal resynchronization, of the duration of QRS (DQRSDc), the occurrence time of the maximum spatial amplitude QRS vector (DMQOT) and of the QT, QRSon to Max of T (QMT) and Max of T to T offset (MTET) time intervals, corrected (DQTccor and DQMTccor) and not corrected (DQTcor, DQMTcor and DMTETcor) for changes in heart rate by the following CAVIAR formulas DQTccor= DQTcor-7.18 $(\sqrt{R R j}-\sqrt{R R i})$ and DQMTccor=DQMTcor7.32 $(\sqrt{R R j}-\sqrt{R R i})$ ); 3) Planarity changes of QRS (Qplan) and T (Rplan); 4) Orientation changes of QRS (OCQ), of its first and last ms (OCI and OCT), of T (OCR), between 
the QRS and T waves (ODQR) and between the first ms of QRS and the T wave (ODIR); 5) Spatial amplitude changes of ST at the J point and at J+60 ms (ST60), of QRS (QRSmax), its first ms (ICMU), of $\mathrm{T}$ (Tmax) and $\mathrm{T}$ corrected for heart rate changes $\left(\mathrm{Tmaxc}=\mathrm{Tmaxc}_{j}-\mathrm{Tmaxc}_{i}\right.$ where $\left.\operatorname{Tmaxc}_{i}=669\left(\operatorname{Tmax}_{i} / 18.9 \sqrt{R R i}+72.6\right)\right)$.

Figure 2 exemplifies the 3D graphical capabilities of the CAVIAR web service, highlighting spatiotemporal QRS and $\mathrm{T}$ changes. 3D plots of superimposed QRS and/or T loops comparisons are displayed in 1, 2, 4, 6 or 9 panels/ page. The user can interactively zoom in/out and rotate each plot, or all plots simultaneously, in any 3D direction with the mouse or with a cursor, and reset the display. Different colours identify the electrical activity before and after the maximum spatial amplitude vector of QRS and T.

\subsection{FR-ECG services composition workflow}

The LYON ECG processing and CAVIAR serial analysis services can be transparently combined in case the provided ECG records do not include a delineated reference beat and the mean RR interval, or if the user prefers to perform CAVIAR analysis on ECGs preprocessed by the LYON program. The LYON + CAVIAR service only provides serial analysis results. But invoking the LYON service alone would provide as explained in 2.1 the 13 unary measurement tables and SCP-ECG files embedding LYON analysis results which could then be used as input of the CAVIAR service.

\section{Results and Discussion}

Table 1 presents some reference data for CAVIAR measurements of QRS and T-wave changes at one month, up to two years interval on about 1000 normal subjects. As also demonstrated by Figure 2, the CAVIAR method allows to outstandingly identify, localize and characterize serial changes in space and time within the QRS and T waves, by overcoming extra-cardiac variability viz recording conditions changes or wave delineation errors. The structural changes in orientation, amplitude and morphology of the electrical activity can thus be precisely characterized as previously demonstrated on an experimental model of 12-lead ECG changes induced by coronary artery occlusion during balloon inflation $[5,6]$. The CAVIAR criteria for ischemia detection yielded a diagnostic accuracy, whatever the location, of respectively 97\% and 98\% (depending on the classification method used), with $98 \%$ sensitivity and 96 to $98 \%$ specificity. In addition, very accurate time interval changes are obtained, at the limit of the sampling time interval [7]. This is an important feature, in particular for the assessment of QT interval changes. We thus expect that the FR-ECG web portal will significantly contribute to the deployment and dissemination of the CAVIAR ECG processing method.
Table 1. CAVIAR serial ECG measurements reference data for normal subjects.

\begin{tabular}{lclc}
\hline Meas. & $\mathbf{m} \pm \mathbf{S D}$ & Meas. & $\mathbf{m} \pm \mathbf{S D}$ \\
\hline QMQD & $56.4 \pm 30.8 \mu \mathrm{V}$ & OCQ & $10.9 \pm 7.8^{\circ}$ \\
\hline IMQD & $13.4 \pm 8.9 \mu \mathrm{V}$ & OCI & $9.5 \pm 7.2^{\circ}$ \\
\hline TMQD & $21.2 \pm 22.9 \mu \mathrm{V}$ & OCT & $13.2 \pm 12.6^{\circ}$ \\
\hline RMQD & $46.1 \pm 31.7 \mu \mathrm{V}$ & OCR & $10.6 \pm 8.7^{\circ}$ \\
\hline DQRSDc & $0.7 \pm 3.9 \mathrm{~ms}$ & IPPAD & $10.3 \pm 12.0^{\circ}$ \\
\hline DMQOT & $-0.1 \pm 3.3 \mathrm{~ms}$ & TPPAD & $5.4 \pm 6.2^{\circ}$ \\
\hline DQTcor & $2.0 \pm 23.4 \mathrm{~ms}$ & ODQR & $-4.9 \pm 15.8^{\circ}$ \\
\hline DQMTcor & $1.9 \pm 23.6 \mathrm{~ms}$ & ODIR & $0.7 \pm 12.8^{\circ}$ \\
\hline DMTETcor & $0.1 \pm 11.0 \mathrm{~ms}$ & ST60 & $-3.6 \pm 33.0 \mu \mathrm{V}$ \\
\hline DQTccor & $-0.2 \pm 14.8 \mathrm{~ms}$ & QRSmax & $40.5 \pm 224.1 \mu \mathrm{V}$ \\
\hline DQMTccor & $-0.3 \pm 14.0 \mathrm{~ms}$ & ICMU & $1.1 \pm 0.1$ \\
\hline QPlan & $0.0 \pm 0.3 \%$ & Tmax & $-6.4 \pm 126.0 \mu \mathrm{V}$ \\
\hline Rplan & $0.0 \pm 0.3 \%$ & Tmaxc & $-14.7 \pm 122.2 \mu \mathrm{V}$ \\
\hline
\end{tabular}

\section{Acknowledgements}

We are deeply grateful to Victor Cojocaru for having set up the FR-ECG web portal and to Joël Placide for the development of the SCP-ECG encoding/decoding tools.

\section{References}

[1] Boothroyd LJ et al. Information on Myocardial Ischemia and Arrhythmias Added by Prehospital Electrocardiograms. Prehospital Emergency Care 2013;17(2):187-92.

[2] ISO 11073-91064:2009 and EN 1064:2005+A1:2007, Medical Informatics-Standard Communication ProtocolComputer assisted Electrocardiography (SCP-ECG).

[3] Rubel P et al. SCP-ECG V3.0: An Enhanced Standard Communication Protocol for Computer-assisted Electrocardiography. Computing in Cardiology 2016;43:309-12.

[4] Arnaud P. et al. Methodology of ECG interpretation in the Lyon program. Methods Inf Med 1990;29(4):393-402.

[5] Fayn J et al. Improvement of the detection of myocardial ischemia thanks to information technologies. Int J Cardiol. 2007;120(2):172-80.

[6] Fayn J. A classification tree approach for cardiac ischemia detection using spatiotemporal information from 3 standard ECG leads. IEEE Trans Biomed Eng. 2011;58(1):95-102.

[7] Fayn J, Rubel P, Mohsen N. An improved method for the precise measurement of serial ECG changes in QRS duration and QT interval. Performance assessment on the CSE noise testing database and on a healthy 720-case-set-population. J Electrocardiol. 1991;24 Suppl:123-7.

Address for correspondence.

Jocelyne FAYN

eTechSanté. Faculté de Médecine Laennec. 7 rue Guillaume Paradin. 69372 LYON cedex 08. France

E-mail jocelyne.fayn@inserm.fr. 